\title{
Design and First Commissioning Results of PLC-based Control Systems at the Mercator Telescope
}

\author{
Wim Pessemier ${ }^{a}$, Geert Deconinck ${ }^{b}$, Gert Raskin $^{a}$, Philippe Saey ${ }^{b, c}$ and Hans Van Winckel $^{a}$ \\ ${ }^{a}$ Institute of Astronomy, KULeuven, Leuven, Belgium; \\ ${ }^{b}$ ESAT - dept. of Electrical Engineering, KULeuven, Leuven, Belgium; \\ ${ }^{c}$ E \& A - Energy \& Automation, KAHO St.-Lieven, Gent, Belgium
}

\begin{abstract}
The $1.2 \mathrm{~m}$ optical Mercator Telescope (based at the Roque de Los Muchachos Observatory at La Palma) is currently in the commissioning phase of a third permanently installed instrument called MAIA (Mercator Advanced Imager for Asteroseismology), a three-channel frame-transfer imager optimized for rapid photometry. Despite having three cryostats, MAIA is designed as a highly compact and portable instrument by using small Stirlingtype cryocoolers, and a single PLC in charge of all temperature control loops, cryocooler interaction, telemetry acquisition and other instrument control related tasks. To accommodate MAIA at the Nasmyth B focal station of the telescope, a new mechanism for the tertiary mirror had to be built since the former mechanism only allowed motor controlled access to the Cassegrain and Nasmyth A focal stations. A second PLC has been installed in order to control the two degrees of freedom of this mirror mechanism by interfacing with its motor controllers, high-precision optical encoders, and limit switches. This PLC is not dedicated to the tertiary mirror control but will serve as a general purpose controller for various tasks related to the telescope and the observatory, as part of a new Telescope Control System primarily based on PLCs and OPC UA communication technology. Due to the central location of the PLC inside the observatory, the position control loops of the mirror mechanism are distributed using EtherCAT as the communication fieldbus. In this paper we present the design and the first commissioning results of both the MAIA instrument control and the tertiary mirror control.
\end{abstract}

Keywords: PLC, Instrument Control, OPC UA, UAF, Model Driven Development

\section{INTRODUCTION}

As the Mercator Advanced Imager for Asteroseismology (MAIA) is currently undergoing its commissioning phase, new scientific opportunities will become available to its community in the coming months. At the time of writing this paper, the beginning of June 2012, MAIA's three 2x3K frame-transfer detectors have just seen first light, 4 years after they were supposed to be launched into space as part of the canceled Eddington ESA mission (see acknowledgments). MAIA allows for simultaneous photometry in the SDSS $u, g$ and $r+i$ bands by using dichroics to split the beam, and additional filters to increase the cut-off accuracy. Wavelengths at the $z$ band are directed towards a commercial grade SBIG camera used for autoguiding.

Since the entire instrument must fit on one of the rotating Nasmyth interfaces of the Mercator Telescope, and since MAIA needs to be portable to other telescopes as well, strong constraints are imposed on the mechanical and electrical designs. MAIA must be compact, must be able to function autonomously and reliably, and must be easy to operate and to maintain. Instead of following the "well-trodden path" involving dedicated temperature controllers and control software written in a popular programming language running on a Linux-equipped PC, we chose an industrial PLC (Programmable Logic Controller) as the main instrument control platform. A Beckhoff CX5020 embedded PC running the TwinCAT PLC software was selected based on the following considerations:

- Hardware dependability: the selected device is rugged and contains no moving parts, and is therefore well adapted to the environmental conditions at the telescope site and to the mechanical stresses imposed by the field derotator.

wim.pessemier@ster.kuleuven.be 


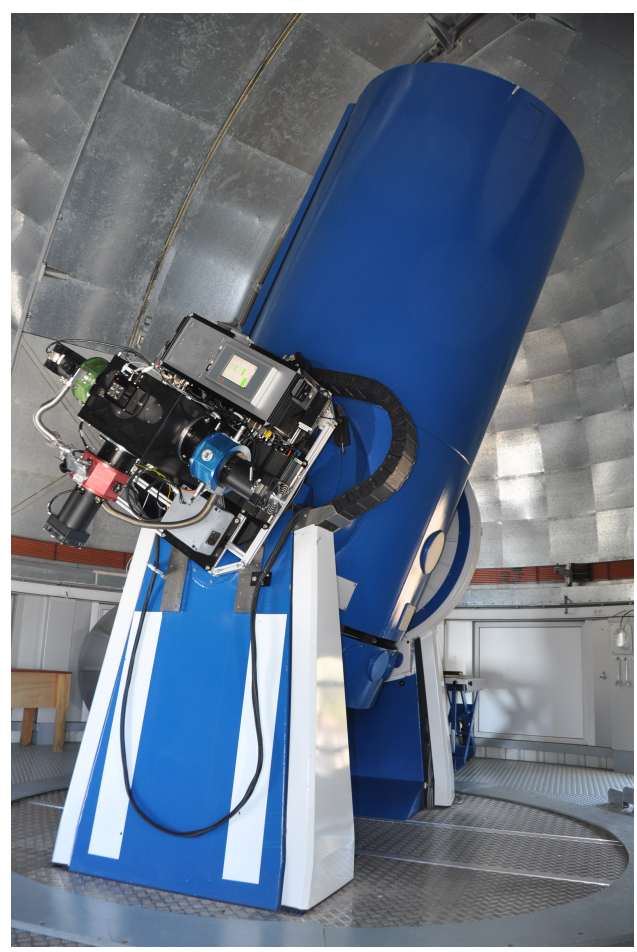

(a) MAIA and the Mercator Telescope

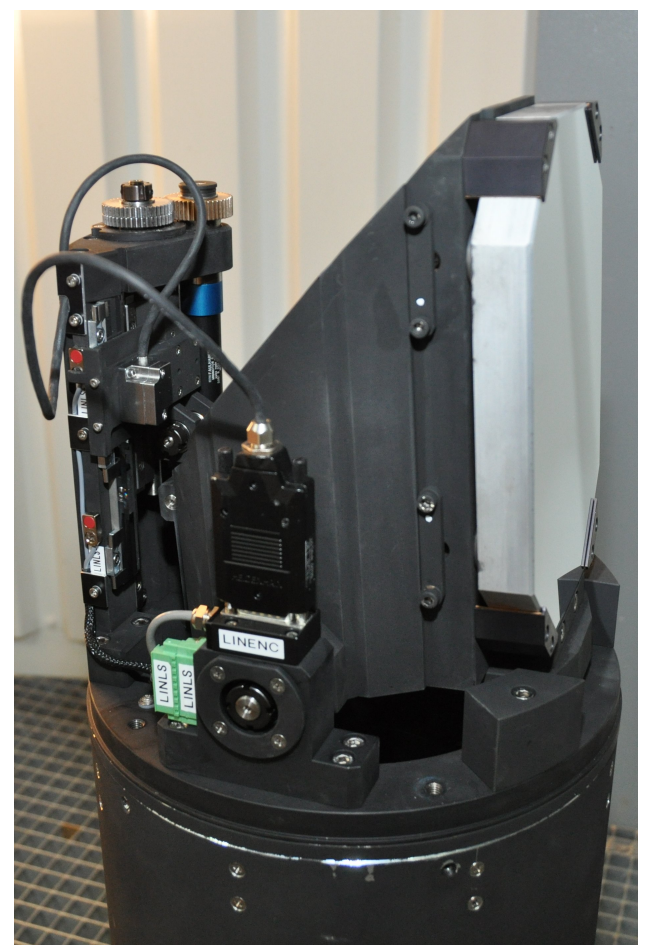

(b) M3

Figure 1. MAIA and M3 during the commissioning phase in May 2012.

- Software dependability: the PLC is programmed in a set of domain-specific languages (standardized as IEC61131-3) which results in very efficient programming, while the PLC run-time system ensures reliable execution of the code in real-time and offers straight-forward debugging to the developer.

- Compactness: the selected PLC is small and can be fit with high-density I/O modules, offering considerable space saving inside the electronics box attached to the instrument.

- Operator-friendliness: a Human Machine Interface (HMI) can easily be programmed and displayed via an industrial touch panel, allowing maintenance and operation without the need for an external PC or even network connection.

- All-in-one solution: the PLC executes the control logic in real-time and offers the remaining CPU time to the operating system, the HMI application, and an OPC UA communication server.

- Cost savings: we estimated the required manpower to be considerably less compared to the "well-trodden path" approach as mentioned before because the PLC comes with an extensive development environment (which allows to develop control and HMI applications in a very short time frame), and because maintenance of the hardware and software platform is effectively outsourced to the PLC manufacturer.

A comparable analysis has led to the decision to control the tertiary mirror (M3) of the Mercator Telescope (and other subsystems in the future) on a similar platform. This mirror was previously only able to be tilted in one degree of freedom, in order to let the beam coming from M2 either pass through to the Cassegrain focal station, or reflect towards one of the Nasmyth focal stations. Since MAIA is installed on the second Nasmyth station, a new mechanical support for M3 had to be developed in order to accommodate the additional degree of freedom. The PLC in control of the motion of M3 will in the future also take care of other telescope subsystems (such as the dome control, the pneumatic support of M1, the hydrostatic bearing of the telescope, etc.) and thus will serve as a "general purpose" controller for the telescope. Because this controller is installed in a cabinet on the ground floor, compactness is not an issue here so we opted for an industrial PC without backplane, running 
the same PLC run-time environment (TwinCAT) as the embedded controller of MAIA. All I/O is connected via the real-time EtherCAT protocol transported by a dedicated ethernet network. Reliability should be similar to the embedded controller of MAIA (as the PC does also not contain moving parts, is also equipped with an Uninterruptible Power Supply (UPS), etc.) but performance is much higher. The increased performance may better accommodate a soon-to-be-released update of the PLC software environment ${ }^{1}$ which may allow us to conveniently compile a $\mathrm{C}++$ pointing kernel on the same platform, execute it in real-time, and distribute its output via the EtherCAT protocol (also in real-time) and via the OPC UA communication server (in non real-time).

\section{HARDWARE OVERVIEW}

Models of the MAIA and M3 control systems are displayed in Fig. 2 and Fig. 3 respectively. Only two kinds of relationships are shown: aggregation, and dependencies between the actuators/sensors and the PLC I/O modules. The latter relationships illustrate the important role of the PLC: nearly all hardware devices related to the control system are directly or indirectly depending on the PLC to fulfill their role. In the next subsections we will describe the depicted hardware components and their relationships to the PLC more in depth.

\subsection{The MAIA control system}

Due to the previously mentioned mechanical constraints, the MAIA instrument has been fit with a densely populated 60x40x30 $\mathrm{cm}$ aluminum box containing most electrical hardware. A careful design was needed to fit all power supplies, cryocooler controllers, the PLC, I/O and some custom electronics in the limited available space while ensuring sufficient airflow and some space for future additional I/O modules or other hardware.

\subsubsection{Power supply and distribution}

A single 230VAC power cable can be plugged into the box in order to power the whole instrument. Due to the limited power consumption of the Stirling coolers, a 24VDC 30 Amps power budget is sufficient to power everything but the CCD controller electronics (requiring a dedicated power supply). Five power supplies in total are installed in the box in order to provide $24 \mathrm{~V}, 12 \mathrm{~V}$ and $6 \mathrm{~V}$ to the various devices. Power distribution, including 230VAC for spare outlets and for the dedicated power supply of the CCD controller electronics, is also fully integrated within the compact aluminum box.

\subsubsection{Instrument control and human machine interfacing}

A Beckhoff CX5020 embedded PC (1.6GHz x86 CPU) with an EtherCAT backplane controls the instrument. In the original design however a more compact and less powerful CX9020 with 533Mhz ARM CPU had been chosen, but this proved to be insufficient to run all software smoothly since we noticed hiccups of the OPC UA server running on the same device. A one-second uninterruptible power supply (UPS) is integrated within the embedded PC in order to avoid data corruption when power is suddenly lost while the PC is writing persistent data to the Compact Flash disk. An industrial touch panel is mounted on the cover of the electronics box and is connected via USB and DVI interfaces to the embedded PC. A small push button is installed just next to the HMI and allows operators to switch on/off the backlight of the HMI on-site. The button is wired to the I/O of the PLC since the latter controls the backlight of the HMI by software (allowing both local and remote control).

\subsubsection{Signal conditioning and shutter electronics}

Two small custom printed circuit boards (PCBs) are also installed in the electronics box. One of them contains the signal conditioning electronics in order to smoothen the electrical current flowing to the CCD heaters, and to divide the voltages of the power supplies in order to be able to measure them with standard 0-10V analog input modules. Another PCB converts the pulses coming from the CCD controller into appropriate power signals for the shutters of the cryostats. 


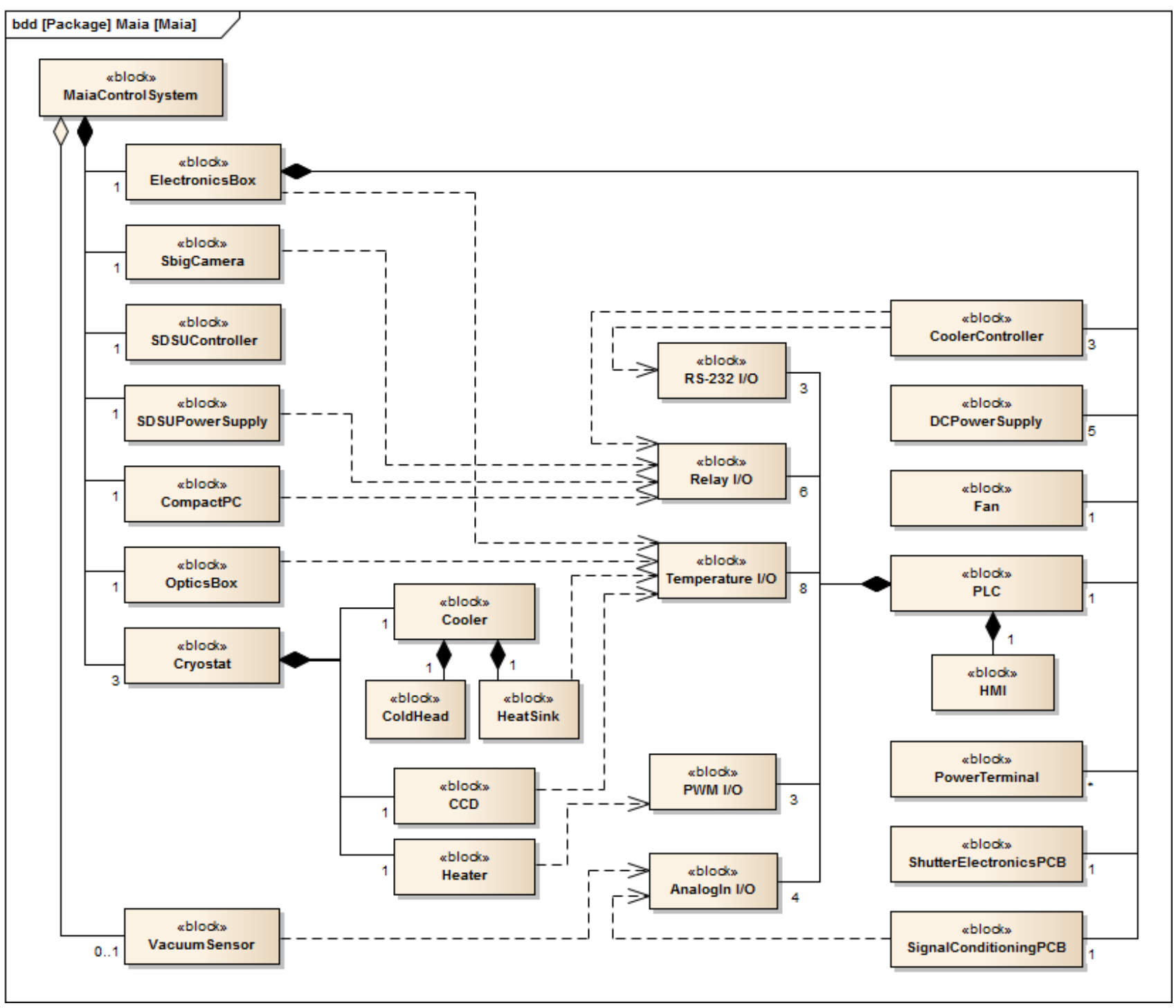

Figure 2. MAIA control system model.

\subsubsection{Cooling and heating}

Due to the previously described challenging mechanical constraints of MAIA, a compact cooling solution with simple mechanical interface was a primary design requirement. Early tests indicated that stacked Peltier elements were only capable of cooling the 2048 x 6144 pixels of the CCD to around $200 \mathrm{~K}$, while Stirling coolers attained a much better result ${ }^{2}(80 \mathrm{~K}$ with an effective cooling power of $5 \mathrm{~W}$ ). The disadvantage of this type of coolers is that they introduce vibrations into the system. At the time of writing this paper, the image degradation caused by these vibrations is still significant, so this mechanical problem must still be solved. Each cryostat has been fitted with a Sunpower CryoTel MT cooler which is connected to the controller electronics of the same manufacturer. The CryoTel controllers are equipped with an RS-232 interface (with simple ASCII protocol) and could therefore easily be interfaced to the PLC by suitable serial I/O modules. Additional inputs of the cooler electronics could be connected conveniently to relay outputs of the PLC I/O in order to switch on/off each controller. We found the CryoTel electronics suitable to stabilize the cold head of the the coolers to approx. $80 \mathrm{~K}+/-0.5$. More accurate temperature stabilization of $+/-0.01 \mathrm{~K}$ of the CCDs around $160 \mathrm{~K}$ is implemented on the PLC by a simple PI control loop. For each cryostat a 16-bit EL3202-0010 I/O module measures the 


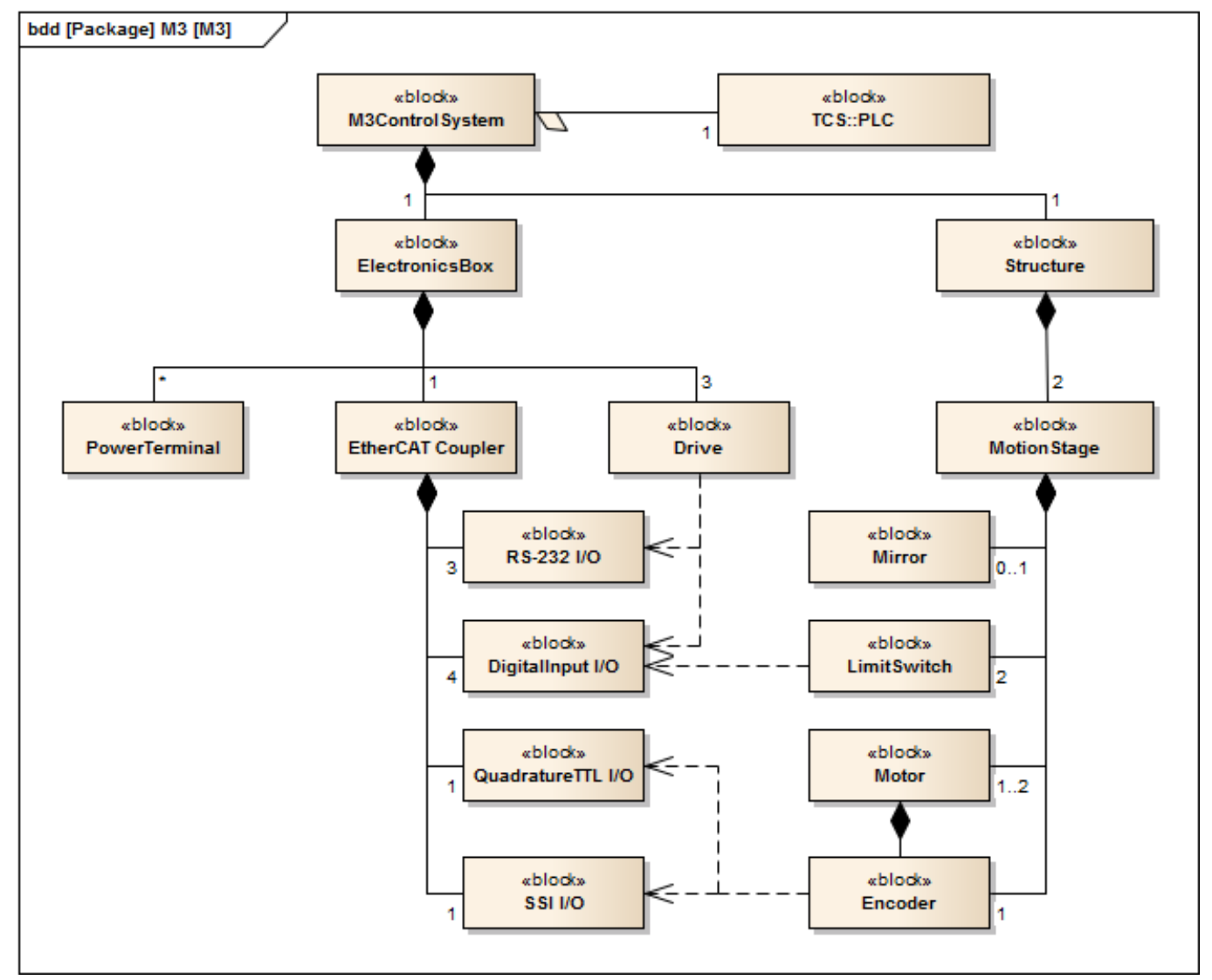

Figure 3. M3 control system model.

CCD temperature, while the EL2502 pulse width modulation (PWM) output module is connected via the signal conditioning electronics to a heating resistor.

\subsubsection{Condition monitoring}

Apart from the cold head temperatures of each cryostat (which are acquired by the CryoTel electronics and can thus easily be queried over the serial interfaces), all other Pt-100 temperature sensors are connected directly to 16-bit EL3202-0010 I/O modules. These include temperature sensors for the CCDs, heat sinks of the Stirling coolers, the optics box and the electronics box. A connector for a Pfeiffer PKR 251 vacuum sensor is also wired to a 16-bit analog input module. This vacuum sensor can thus be connected to the PLC without any interfacing electronics, and its output can be displayed and logged as soon as the PLC senses its presence.

\subsubsection{Scientific and guiding data acquisition}

A small fanless industrial PC is also attached to the instrument. It hosts the standard environment of the Mercator Observatory Control System (MOCS) ${ }^{3}$ on a SuSE Linux operating system (soon to be replaced by Scientific Linux). While the $\mathrm{UAF}^{4}$ or Unified Automation Framework already provides an OPC UA client interface to the MOCS, it does not yet include OPC UA server functionality. This means that the detector software interface is currently still exposed to the network via the custom encoding and information model defined by the MOCS framework, instead of an OPC UA interface (which includes a meta-model, standardized data access and alarming, support for name spaces, etc.).

\subsection{The M3 control system}

Even though the number of involved electric components is limited, we found the M3 control system a particularly good test case for future PLC-based motion control solutions at the Mercator Telescope because: 


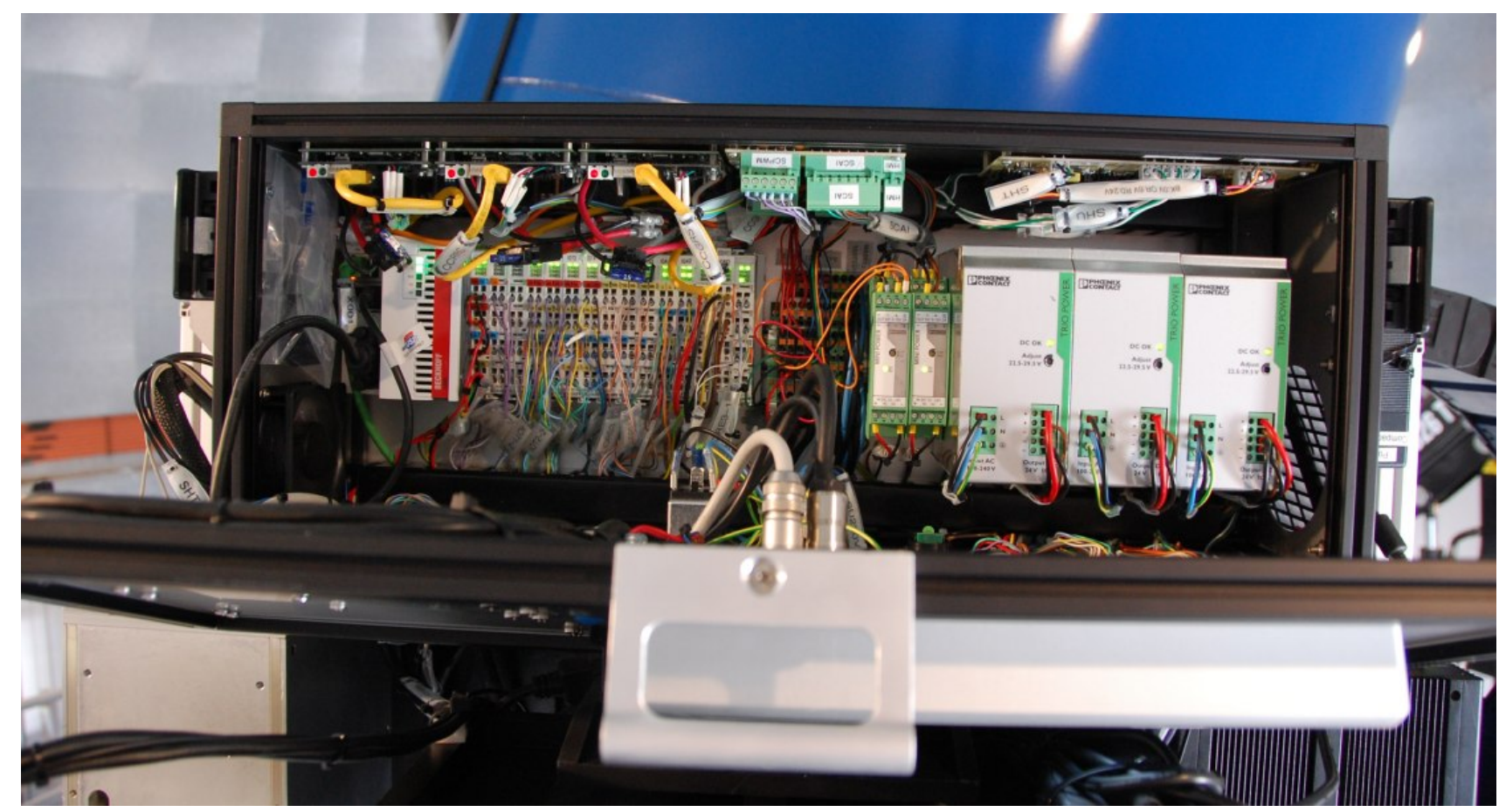

Figure 4. Densely populated (but all inclusive) control electronics box of MAIA.

- the required absolute accuracy of the mirror support is quite high (see further);

- interaction is needed between a master motor and a slave anti-backlash motor;

- a variety of hardware is used such as an absolute and an incremental encoder, and a translating and a rotating mechanism;

- despite the proprietary serial protocol of the miniature drives, a full PLCopen compliant software solution could be developed.

The software in control of M3 is running on a Beckhoff C6325 industrial "cabinet" PC, which is installed in one of the electronics cabinets located centrally in the telescope building. This PC is larger, heavier and more powerful than the embedded PC of M3, but similarly it does not contain moving parts since it is cooled passively and it contains a Compact Flash disk instead of a classic hard drive. The PC is connected via an industrial grade ethernet network to a small electronics box attached to the telescope, close to the tertiary mirror. The network passes a few EtherCAT junction points in order to be able to easily branch off more EtherCAT segments in the future.

\subsubsection{Translation stage}

The translation stage contains a worm drive and a Heidenhain LIP471 linear encoder with an absolute accuracy of $+/-1 \mu \mathrm{m}$. This encoder has built-in interpolation and digitizing electronics and can therefore directly be connected to a Beckhoff EL5101 encoder interface. A single home-mark in the middle of the encoder range is available for absolute referencing via the same interface, but during the commissioning phase we were unable to have the encoder electronics produce the homing pulse (possibly because we did not have access to a suitable encoder calibration device from Heidenhain). While this problem remains to be solved, we currently reference the encoder by having the worm gear pass the electrical limit switches and run slowly into the mechanical stop. Repeatability proved to be better than $1 \mu \mathrm{m}$, and there is no risk for mechanical damage since the current limit of the motor is lowered during the initialization algorithm, and since an additional mechanical slip coupling protects 


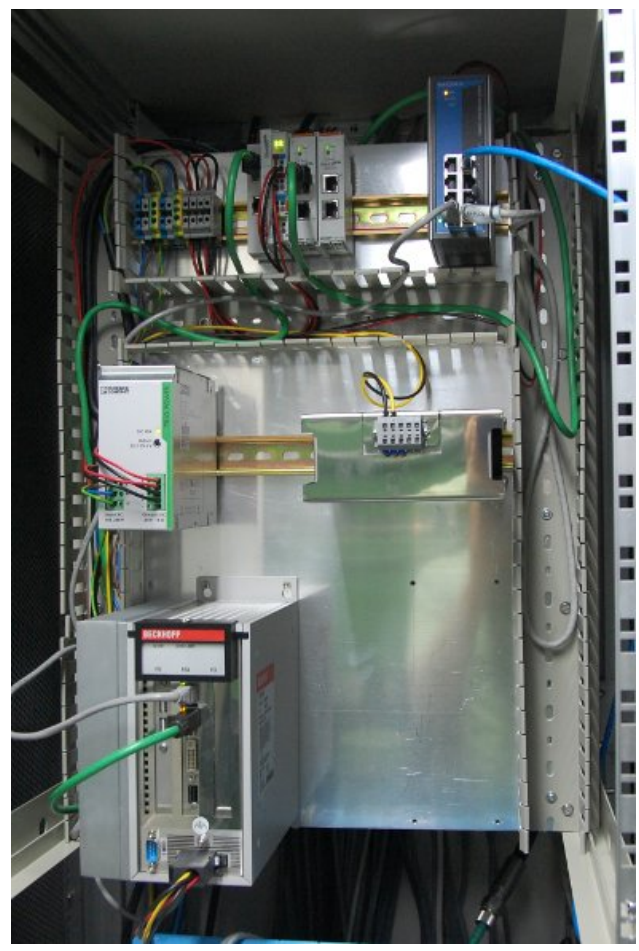

(a) One of the TCS electronics racks

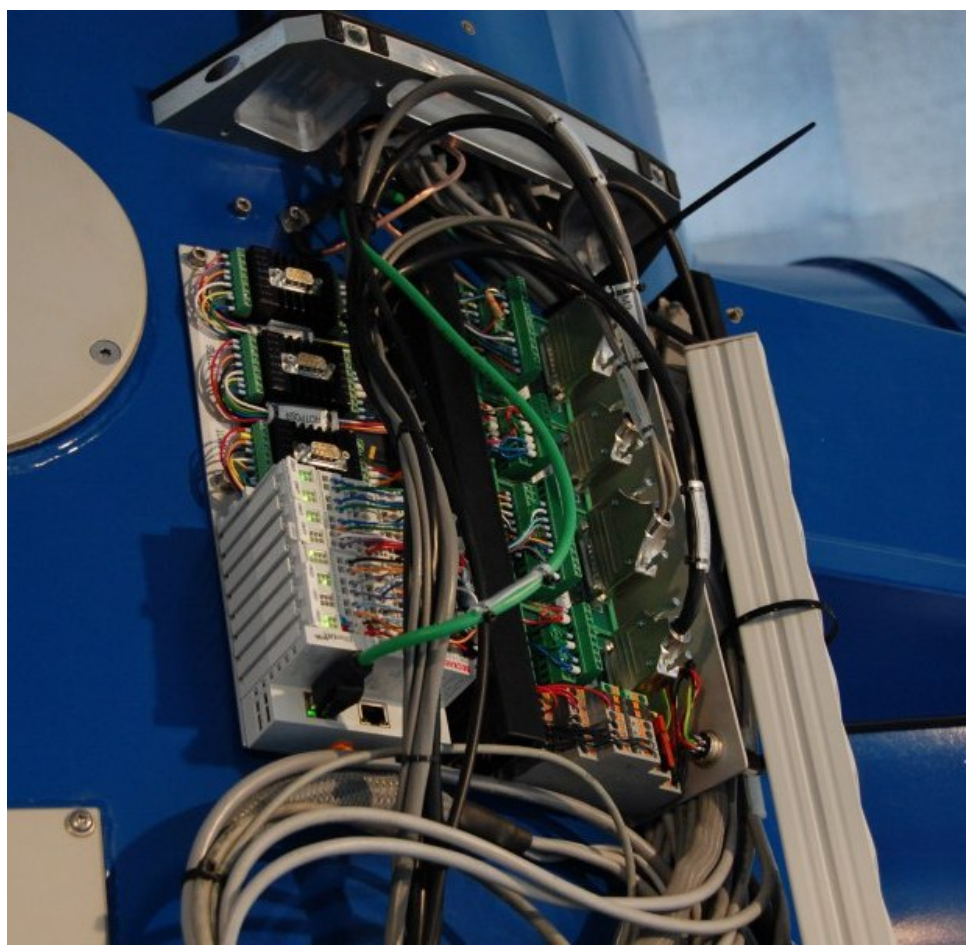

(b) M3 electronics connected by EtherCAT

Figure 5. M3 electronics during the commissioning phase in May 2012.

the mechanism. Due to the sufficiently high accuracy and non-reversibility of the worm drive, no anti-backlash measures have to be taken for the translation stage, so a single Faulhaber 2057S synchronous servomotor can drive the whole mechanism. The motor is controlled by a Faulhaber MCBL3003/S motion controller, which is interfaced to the PLC via RS-232 I/O modules.

\subsubsection{Rotation stage}

The rotation stage carrying the mirror holder consists of a large gear wheel driven by two Faulhaber 3564 synchronous servomotors, each of them equipped with a large irreversible 1:1600 planetary reduction. One of the motors is responsible for the precise positioning and is therefore connected directly to the large gear. The other motor is connected via a magnetic clutch in order to provide a constant torque (thereby eliminating the backlash) even when the drives are powered off. A Kübler Sendix F3663 absolute encoder with an absolute accuracy of 33 arcseconds (according to the manufacturer) is used for accurately positioning the rotation stage. This encoder is continuously read by the PLC via the Synchronous Serial Interface (SSI) protocol.

\section{SOFTWARE}

As advocated by our paper on the Unified Architecture Framework, ${ }^{4}$ we believe that the first and foremost requirement for the PLC software of MAIA and M3 is its capability to be integrated into the higher level Mercator Observatory Control System in a consistent, future-proof and straight-forward way. OPC UA plays an important role in that quest because it supports a rich data model and because client and server implementations are available for many platforms, including PLCs. Furthermore, the growing support from industry implies that more OPC UA enabled COTS products (such as HMI or SCADA software) may become available in the future, thereby expanding the available range of interoperable products.

In order to expose the address space of the MAIA and M3 PLCs, we therefore opted to install the TwinCAT OPC UA server software package on each of them. This effectively converts each PLC with nearly no effort into a full blown OPC UA server offering standardized data access, secure communication, a meta-model, etc. The 
OPC UA server automatically maps programs, function blocks and variables to their standard representation as defined by the OPC UA specifications. ${ }^{5}$

\subsection{Structural controller code}

Since the data model as exposed by the OPC UA server is tightly coupled to the structure of the IEC61131-3 application code, we need to be careful not to model our software too much to the PLC platform particularities. Doing so would violate our goal to create an evolvable control system that can easily be integrated into the higher level system. Instead, we have developed a methodology to apply a much more generic and "OPC UA friendly" model-driven development approach to the (non object-oriented and cyclically executed) IEC611313 PLC software. This methodology is described in Appendix A. It involves the transformation of a Platform Independent Model (PIM) to a Platform Specific Model (PSM) according to a custom defined mapping. The same CASE (Computer Aided Software Engineering) tool that performed the transformation can then automatically generate IEC61131-3 compliant code, and finally the PLC development environment can import this code and expose the PSM via OPC UA. Since the conversion from the PIM to the PSM is reversible, a client (or an intermediary server) can perform this inverse mapping on-the-fly and thereby interact transparently with the PIM. While we have not (yet) developed such a client with the reverse mapping implementation, we do have a working implementation of the forward transformation from PIM to PSM, and the associated IEC61131-3 code generation. We have conveniently implemented this transformation and code-generation using the template editor of Enterprise Architect by Sparx Systems. The PIMs that we have developed for MAIA and M3 are shown in Fig. 7 and 8 respectively. The resulting PSM for the tertiary mirror control as viewed by a generic OPC UA client, can be seen in Fig. 10.

The methodology as mentioned in the previous paragraph is based on the principle that structural code and behavioral code can cleanly be separated by applying the so-called "visitor" paradigm. Because the structural code generated from the PSM is not well suited to include the behavioral code, it is up to the application programmer to create functional code that "visits" the structural code during each PLC scan in order to perform its role. This behavioral code will be elaborated in the next section.

\subsection{Behavioral controller code}

Most of the "visiting" behavioral code for MAIA and M3 is written in Ladder Diagram (LD), Sequential Function Chart (SFC) and Structured Text (ST). Besides the standard function block library as provided by the PLC programming environment, we also required an additional library for the RS-232 communication to the CryoTel cryocooler controllers (in the case of MAIA) and the Faulhaber miniature drives (in the case of M3). We also obtained the TwinCAT Controller Toolbox in order to conveniently control the MAIA CCD temperatures (via PI controller function blocks) and to filter sensor signals (via appropriate moving average function blocks).

As can be seen on the PIMs of Fig. 7 and 8, the configuration and initialization interfaces of MAIA and M3 are very similar. Three configurations are assigned to each subsystem: a currently active configuration (currentConfiguration), a locally stored configuration (localConfiguration) and a remotely editable configuration (remoteConfiguration). The local configuration is stored persistently on the PLC (for instance via the RETAINED keyword of IEC61131-3) and is copied to the currently active configuration at start up. Depending on the contents of the configuration, the subsystem may already start to perform several tasks if needed, even before an initialization command is given. For instance, MAIA is configured to start the temperature control loops automatically after a reboot (e.g. induced by a power failure), while M3 is configured to wait for the initialization command before the homing of the axes takes place. In order to modify the currently active configuration externally (e.g. via OPC UA), one may modify this configuration directly, or indirectly via the remoteConfiguration. The latter will only take effect as soon as the loadRemoteConfiguration() operation is called, in order to allow a consistent modification of several configuration parameters at once.

While the TwinCAT PLC environment is sufficient to control MAIA, for M3 we chose to use the TwinCAT NC PTP (Numerical Control, Point-To-Point) edition. This expansion of TwinCAT PLC includes several software modules to control the positioning of axes. Even though our Faulhaber motor controllers were not supported out-of-the-box by TwinCAT, we were able to setup the basic communication between the NC interface and the motor controllers. The result is that we were able to conveniently commission the axes using the TwinCAT 
built-in front-end for axis control, and that we could develop our motion control software using the standard PLCopen motion control function blocks. More advanced motion profiles were therefore particularly easy to implement. For instance, to put a minimal strain on the rotation stage gear mechanism during positioning, we make sure the anti-backlash torque is first lifted (by moving only the anti-backlash motor equipped with the magnetic clutch), then both motors are coupled in a master-slave configuration and are moved synchronously, and afterwards the anti-backlash torque is restored and the precise positioning is performed. Since the drives are configured in velocity control mode and the position loops are closed by the NC task, much freedom is given to the PLC application developer to configure and control the axes.

\subsection{Higher level software integration}

Integration of the new PLC systems into the Mercator Observatory Control Software (MOCS) is currently still limited since MAIA and M3 do not require much interaction, and since we're in the process of assessing a commercial software package (WinCC OA by Siemens/ETM) to become the main SCADA system for our telescope control system. This commercial software package supports OPC UA and thus offers a nearly "plugand-play" interface to our PLC back-ends. Until the assessment is finished, we control M3 with a provisional GUI which has been quickly developed using Python/Qt and UAF, ${ }^{4}$ the Unified Architecture Framework. The latter software package we have developed in-house as an abstraction layer between a commercial OPC UA Software Development Kit (SDK) and user applications written in $\mathrm{C}++$ and Python. The UAF takes care of many technical concerns, which allowed us to quickly and with minimal effort create a graphical OPC UA client for controlling M3, and a non-graphical OPC UA client to read telemetry data from MAIA and store this data in the central database of the Mercator observatory. Both clients use the simplified Python API of UAF to create monitored items on the PLC side, and therefore benefit from the efficient asynchronous data delivery defined by OPC UA. Currently only the OPC UA client side is supported by UAF, but this is sufficient for controlling M3 and reading telemetry data from MAIA because the OPC UA server side is running directly on the PLCs. Until we have finished the assessment of WinCC OA, we control MAIA by simply capturing the screen of the TwinCAT HMI via a free graphical desktop-sharing application running on Scientific Linux via Windows emulation software. Both the M3 Python/Qt/UAF application and the MAIA HMI can be seen in Fig. 9.

\section{CONCLUSIONS}

In this paper we presented the hardware and software solutions for the control of the MAIA instrument and the tertiary mirror support of the Mercator telescope. Since the end of May 2012, both control systems have been commissioned and are now fully operational at the observatory at La Palma. While their stability remains to be evaluated in the coming months, we expect little or no problems with these systems since they have proven to work very reliably for the last months already during the integration tests in Belgium. We are convinced that the choice for PLCs over the "well-trodden path" of Linux PCs and software written in popular programming languages, already helped us to deliver a reliable, economical, and time-saving solution. In the future we expect to gain even more due to the inherent dependability and maintainability characteristics of these industrial devices. A cornerstone in our setup is the choice for OPC UA as the means to integrate the MAIA and M3 control systems into the observatory-wide control system, because it allows us to expose a rich information model in a platform-independent way to both custom clients (via UAF, the Unified Architecture Framework) and OPC UA enabled COTS client software packages.

\section{ACKNOWLEDGMENTS}

The development of this system has received funding from the European Research Council under the European Community's Seventh Framework Programme (FP7/2007-2013)/ERC grant agreement n 227224 (PROSPERITY) and from the Fund for Scientific Research of Flanders (G.0332.06). 


\section{APPENDIX A. MODEL-DRIVEN DEVELOPMENT OF IEC61131-3 SOFTWARE}

PLC software is based on a different programming model compared to object-oriented software as commonly written in languages such as $\mathrm{C}++$, Python or Java. First of all, PLC software is executed as part of a cycle typically consisting of four phases: operating system tasks (including the handling of remote communication), a scan of the inputs (sensor values and those set by external communication), the execution of the user program logic, and an output scan to copy the updated values of the process image to the I/O modules and to the operating system. Secondly, PLC software according to the mainstream IEC61131-3 standard is based on three kinds of socalled Program Organization Units (POU): Programs (PRG), Function Blocks (FB) and Functions (F). Programs are executed cyclically and are made up of stateless Functions and stateful Function Block instances, which are connected to each other via inputs and outputs.

While Function blocks share some of the characteristics of object-oriented "objects", they are inherently different, which means that a model-driven design methodology is difficult to apply to IEC61131-3 projects. A number of papers exist that try to work around this problem, ${ }^{6}$ but to our knowledge none of them considers how the resulting address space will look like when exposed to the network, in particular via OPC UA. Since the integration of PLCs into the higher level system is of primary importance to us, it is our goal to expose a clean "object-oriented-like" model down to the PLC level. This implies that the software running on the PLC should be structured similarly to the object hierarchy it tries to represent

While the behavior of Function Blocks clearly differs from behavior of classes in object-oriented design, their structural characteristics (i.e. their outside appearance) is actually very similar. Both entities can be instantiated, and contain data members that can be private or public. From the same point of view, functions are also structurally similar to Function Blocks since both contain input and output parameters, and both can be members of classes resp. other Function Blocks. So apart from their behavioral aspects, a simple mapping can be developed between them.

The behavioral differences between object-oriented classes and methods on the one hand, and Function Blocks on the other hand, are more difficult to resolve. Interestingly though, the behavior of these entities does not have to be tied to the entities themselves. According to the "visitor pattern", ${ }^{7}$ an algorithm may be decoupled from the object structure on which it operates. In our case, we can map classes and methods to Function Blocks, while implementing the behavior of the classes and methods inside other externally "visiting" Function Blocks.

This is exemplified in Fig. 6. As one can see, all classes and functions from the object-oriented model are transformed into a structurally similar IEC61131-3 model. The latter model can then be exported easily to IEC61131-3 code, which can be imported into the PLC programming environment.

Also noticeable in the Fig. 6 is the addition of variables such as _objCmd, metCmd, TRIGGER, etc.. These members are not part of the source model, but have been added by the model-to-model transformation. They are included because the structural code not only requires additional functional behavior (via visiting external Function Blocks) but also technical behavior. For instance, Function Blocks that mimic methods can be "called" by first changing their input parameters and then setting their TRIGGER flag to True. The PLC can now check this trigger each cycle, and modify the diagnostics information in order to notify the visiting Function Blocks that implement the functional concerns. Since the PLC has to repetitively and recursively execute this technical behavior, we decided to include it in the automatically generated structural code.

Due to the "industrial" nature of the control system, software alarms are also considered as one of the technical concerns, which explains why their handling code is also produced by the code generator. This means that the PLC can recursively update all alarms at the start and the end of each execution cycle. These alarms are modeled by transforming the OPC UA Alarms information model (including alarm objects with methods) into our previously described IEC61131-3 model. Some PLCs (including our Beckhoff ones) also support standard OPC UA alarms, but since we prefer to have full control over the alarms, we have chosen to expose them as Function Blocks. As can be seen in the PIMs of MAIA and M3, the code generator interprets the standard OPC UA HasCondition relationship to inform the PLC which variable can trigger the alarm. 


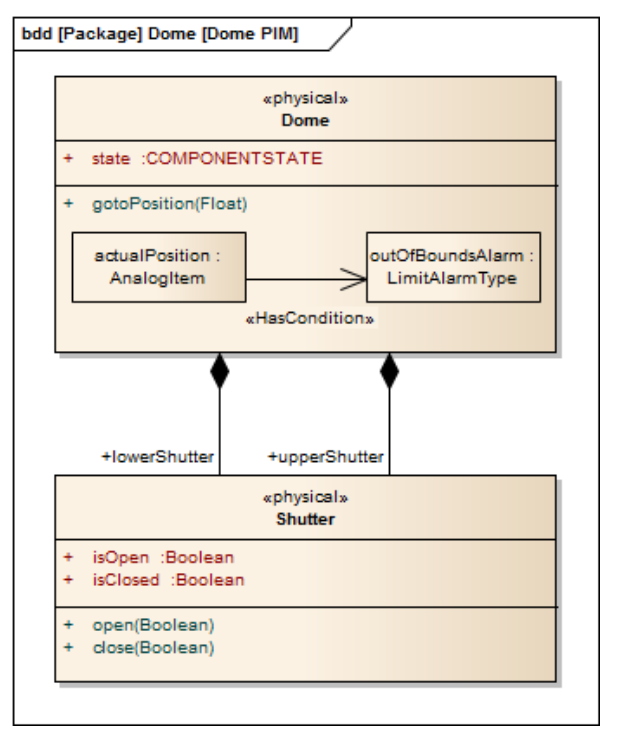

(a) PIM

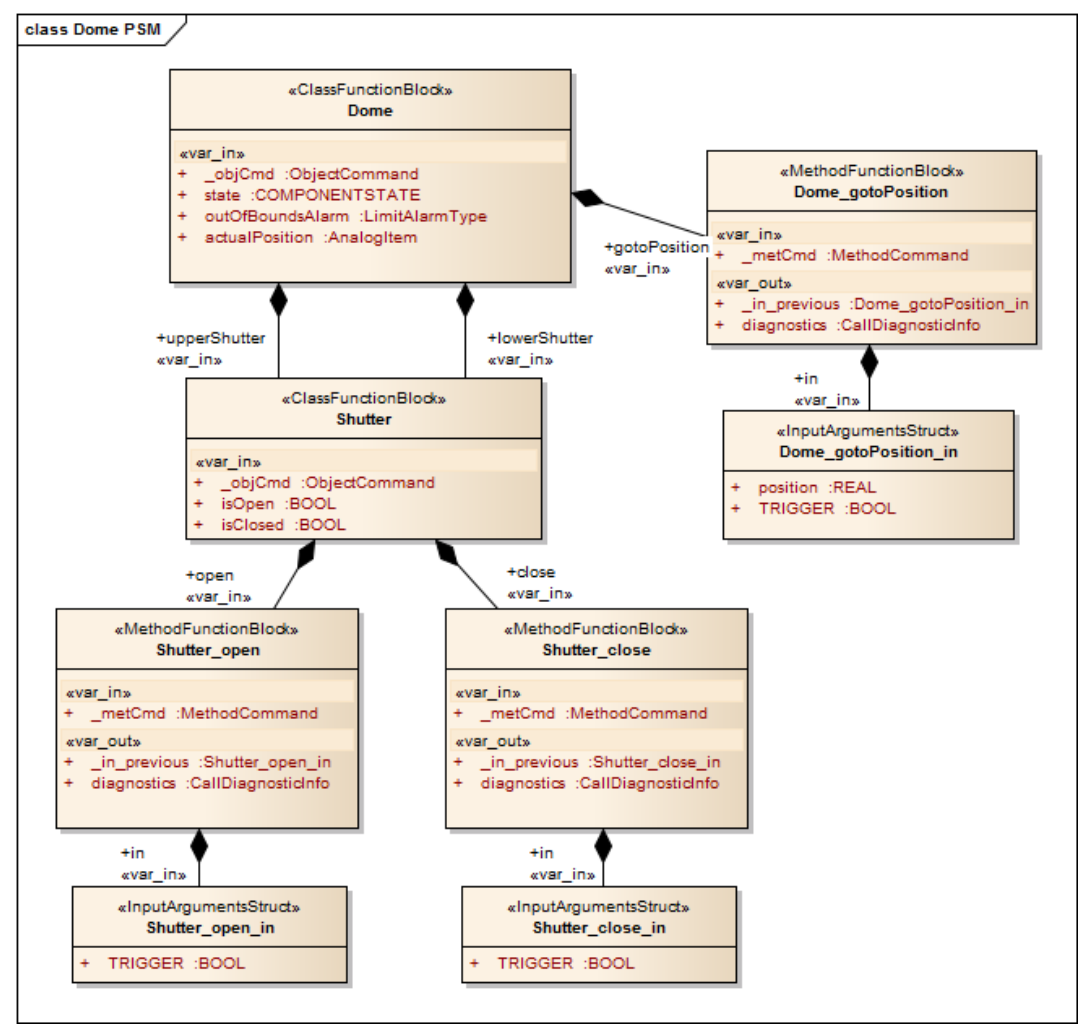

(b) Corresponding PSM

Figure 6. Example of a transformation from a PIM to an IEC61131-3 PSM.

\section{REFERENCES}

[1] Beckhoff, "Twincat 3 - extended automation," (2012). http://www.beckhoff.com/english/twincat/ twincat-3.htm.

[2] Vandersteen, J., Raskin, G., Aerts, C., Bloemen, S., Merges, F., Morren, J., stensen, R. H., Padilla, J. P., Pessemier, W., Prins, S., Swevers, J., and Van Winckel, H., "Maia - the mercator advanced imager for asteroseismology: performance verification and first test result," in [Ground-based and Airborne Instrumentation for Astronomy IV], Proc. SPIE 8446 (2012).

[3] Pessemier, W., Raskin, G., Prins, S., Merges, F., Padilla, J. P., Van Winckel, H., and Waelkens, C., "Towards a new mercator observatory control system," in [Software and Cyberinfrastructure for Astronomy], Proc. SPIE 7740, 77403B (2010).

[4] Pessemier, W., Deconinck, G., Raskin, G., Saey, P., and Van Winckel, H., "Uaf: a generic opc unified architecture framework," in [Software and Cyberinfrastructure for Astronomy II: Technology Advancements], Proc. SPIE 8451 (2012).

[5] OPC Foundation and PLCopen, "OPC UA Information Model for IEC 61131-3, v1.00," (2010).

[6] Vogel-Heuser, B., Witsch, D., and Katzke, U., "Automatic code generation from a uml model to iec 61131-3 and system configuration tools," International Conference on Control and Automation 2, 1034 - 1039 (2005).

[7] Gamma, E., Helm, R., Johnson, R., and Vlissides, J., [Design Patterns: Elements of Reusable Object-Oriented Software], Addison-Wesley (1994). 


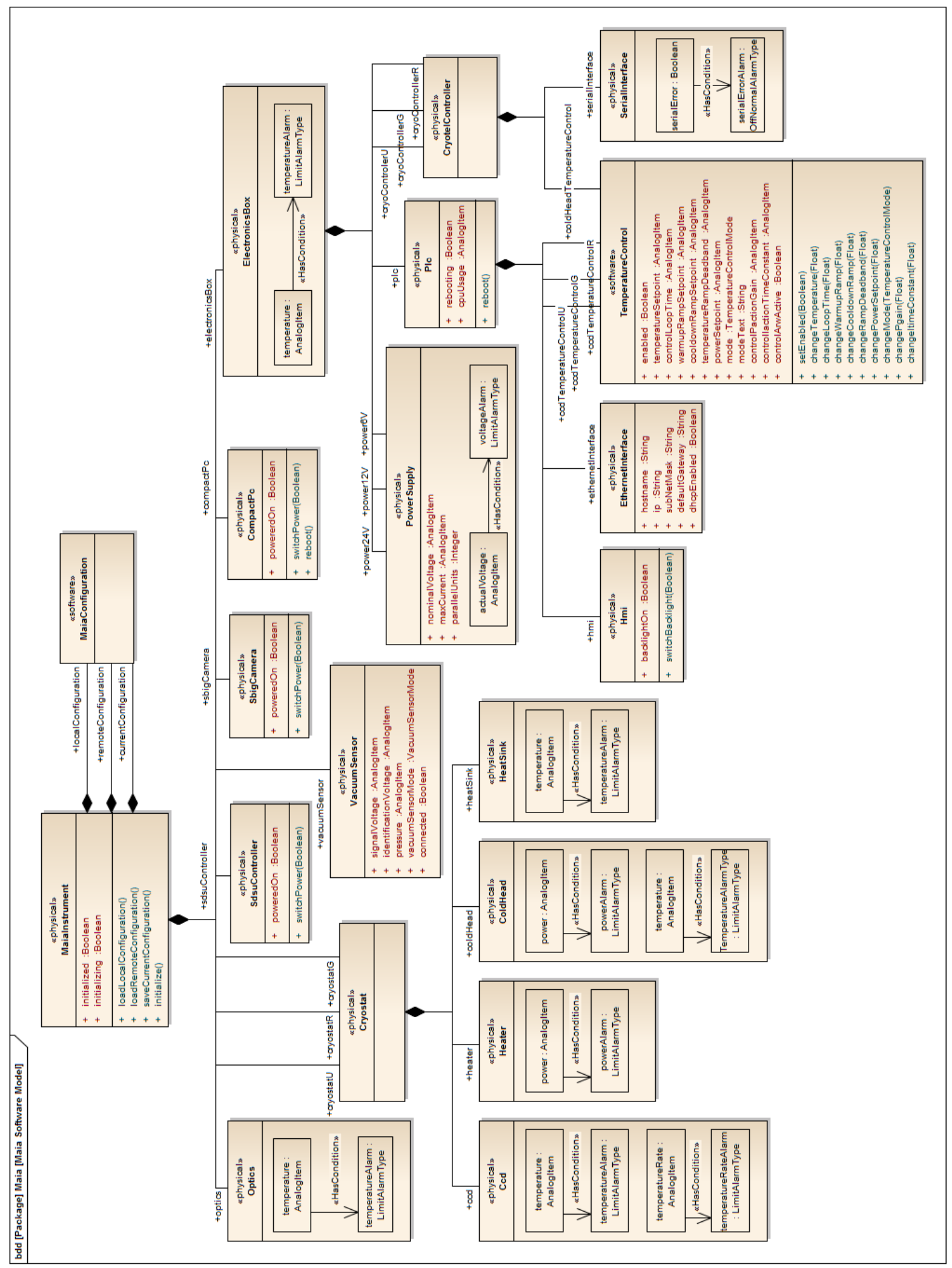

Figure 7. MAIA software model. 


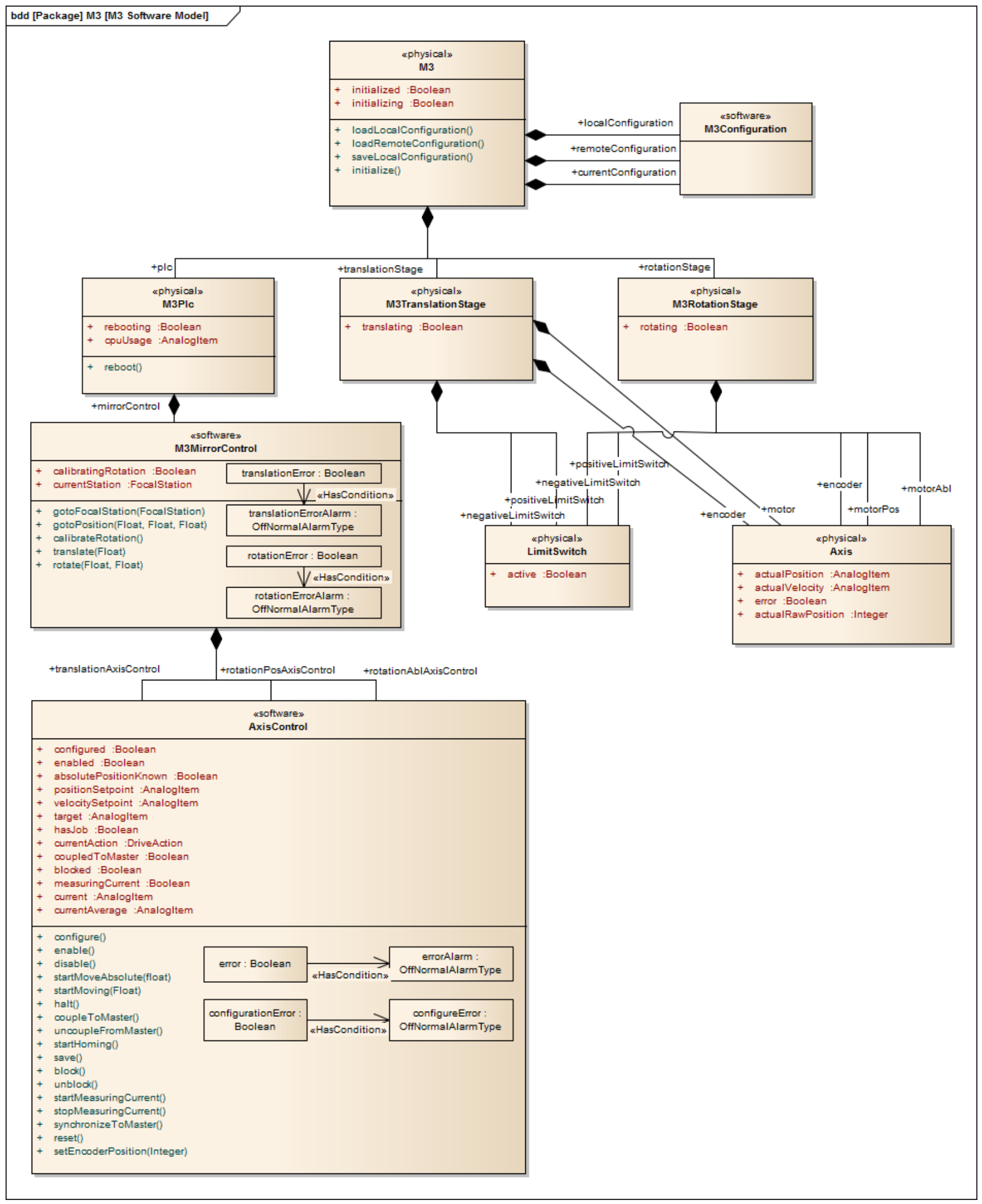

Figure 8. M3 software model. 


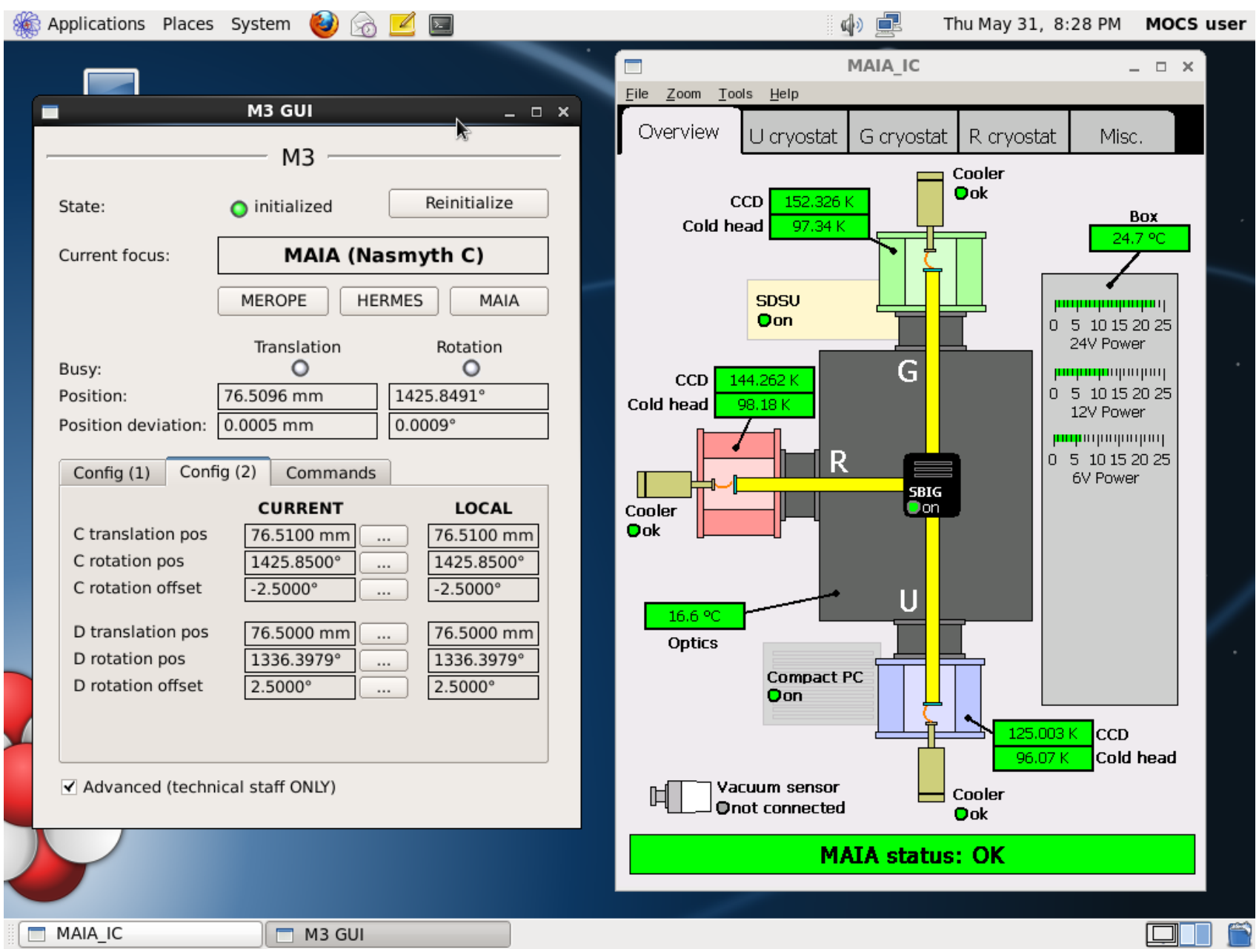

Figure 9. OPC UA client for M3 (left) and screen forwarding of the MAIA HMI (right). 


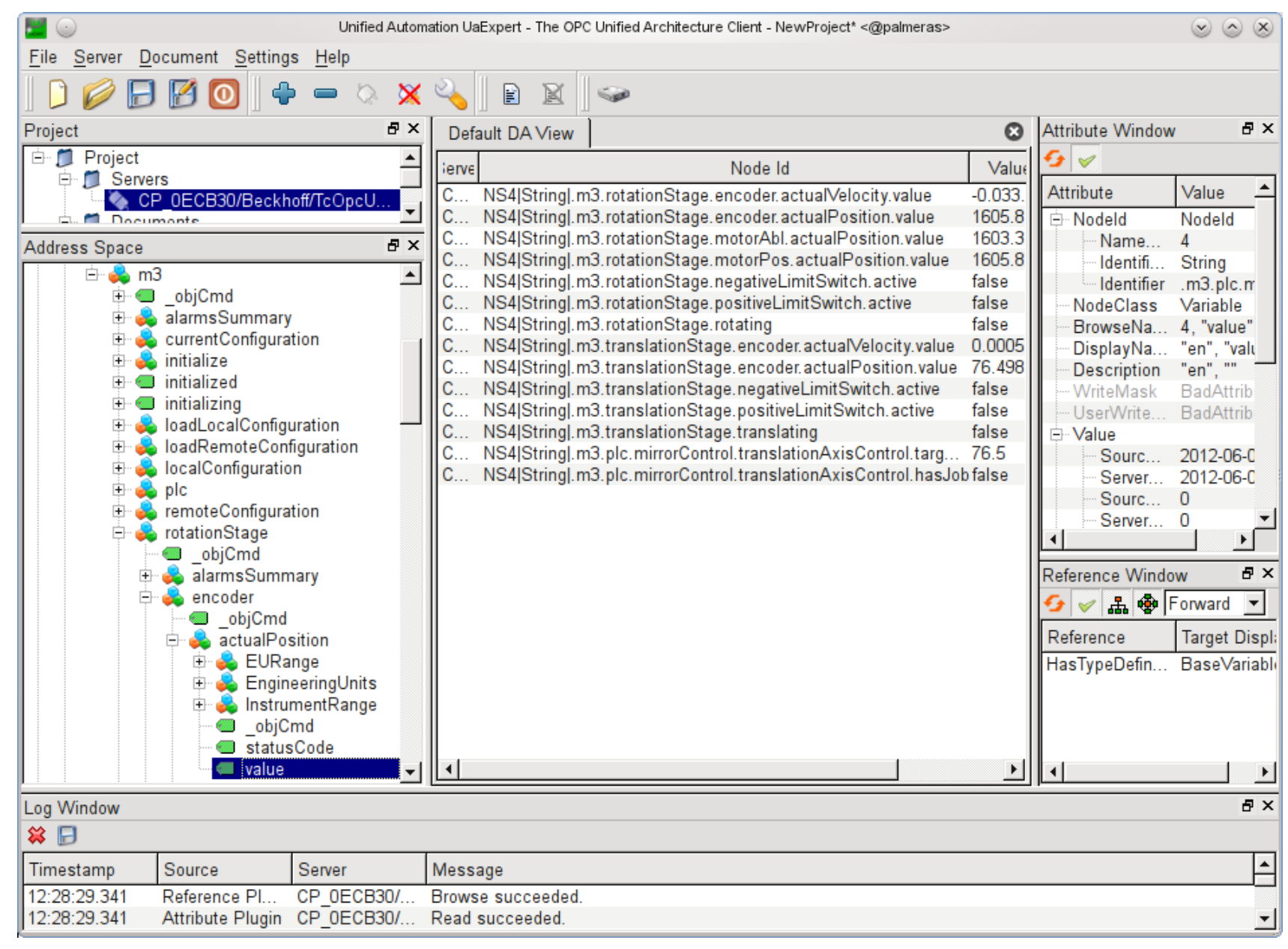

Figure 10. Structured address space of the M3 control system. 